\title{
Cyclotron resonant interactions in cosmic particle accelerators
}

\author{
Toshio Terasawa - Shuichi Matsukiyo
}

Received: 10 March 2012 / Accepted: 27 March 2012

\begin{abstract}
A review is given for cyclotron resonant interactions in space plasmas. After giving a simple formulation for the test particle approach, illustrative examples for resonant interactions are given. It is shown that for obliquely propagating whistler waves, not only fundamental cyclotron resonance, but also other resonances, such as transittime resonance, anomalous cyclotron resonance, higher-harmonic cyclotron resonance, and even subharmonic resonance can come into play. A few recent topics of cyclotron resonant interactions, such as electron injection in shocks, cyclotron resonant heating of solar wind heavy ions, and relativistic modifications, are also reviewed.
\end{abstract}

Keywords particle acceleration · plasma turbulence $\cdot$ cyclotron resonance

\section{Introduction}

It is generally believed that for efficient nonthermal particle acceleration in various explosive cosmic events strong plasma turbulence play the essential role. For nonthermal particles to emerge from the pools of majority thermal particle populations, some selection mechanism(s) should work in turbulent environments. The most likely selection mechanism is due to resonance interaction which assures the efficient energy/momentum transfer from the turbulence to nonthermal particles. In this review, we will discuss the cyclotron (and transit-time) resonance interactions which would be viable in various space plasma environments.

T. Terasawa

Institute for Cosmic Ray Research, University of Tokyo, 5-1-5 Kashiwa-no-ha, Kashiwa city, Chiba 277-8582 Japan

Tel.:+81-4-7136-5173

Fax:+81-4-7136-3194

E-mail: terasawa@icrr.u-tokyo.ac.jp

S. Matsukiyo

Earth System Science and Technology, Kyushu University, Kasuga city, Fukuoka 816-8580, Japan 
In usual space-plasma environments, the electromagnetic fields have the background components $\left(\mathbf{E}_{0}, \mathbf{B}_{0}\right)$ and overlapping multi-wave components, $\left(\mathbf{E}_{w}^{\#}, \mathbf{B}_{w}^{\#}\right)$ where $\#=$ $1,2, \ldots$ are the mode ID numbers. The generalized Ohm's law gives the relation between $\mathbf{E}_{0}$ and $\mathbf{B}_{0}$ as

$$
\mathbf{E}_{0}=-(1 / c) \mathbf{V}_{\text {bulk }} \times \mathbf{B}_{0},
$$

where we write the bulk velocity of the plasma as $\mathbf{V}_{\text {bulk }}$ and neglect higher order terms caused by the spatial variations of $\mathbf{B}_{0}$ and electron thermal pressure. If we make the coordinate transformation to the rest frame of the bulk plasma, $\mathbf{E}_{0}$ vanishes. For a nonrelativistic electron, the equation of motion is 1

$$
\begin{aligned}
& \frac{d \mathbf{V}_{e}}{d t}+\Omega_{c e} \mathbf{V}_{e} \times \frac{\mathbf{B}_{0}}{\left|\mathbf{B}_{0}\right|}=-\frac{e}{m_{e}} \sum_{\#}\left\{\mathbf{E}_{w}^{\#}+\frac{1}{c} \mathbf{V}_{e} \times \mathbf{B}_{w}^{\#}\right\} \\
& \frac{d \mathbf{r}_{e}}{d t} \quad=\quad \mathbf{V}_{e}
\end{aligned}
$$

where $\Omega_{c e}$ is the electron cyclotron frequency $e B_{0} / m_{e} c$. (1) can be regarded as an oscillator equation of the eigen-frequency $\Omega_{c e}$ with the forcing term on the right hand side. When we integrate (1), we evaluate $\mathbf{E}_{w}^{\#}$ and $\mathbf{B}_{w}^{\#}$ at the electron position $\mathbf{r}_{e}(t)$ which is affected by the existence of the waves. Therefore the system of equations (1) and (2) have nonlinearity, from which mode coupling effects among the wave components can appear. However, if the amplitudes of waves are small enough, we can separately integrate (1) for each wave,

$$
\frac{d \mathbf{V}_{e}}{d t}+\Omega_{c e} \mathbf{V}_{e} \times \frac{\mathbf{B}_{0}}{\left|\mathbf{B}_{0}\right|}=-\frac{e}{m_{e}}\left\{\mathbf{E}_{w}^{\#}+\frac{1}{c} \mathbf{V}_{e} \times \mathbf{B}_{w}^{\#}\right\}
$$

and then superpose the results afterward (the standard procedure in the quasi-linear treatment). From here we choose a single wave mode and drop the superfix \# $\mathrm{L}^{2}$.

For simplicity we consider electromagnetic waves in a cold uniform plasma consisting of electrons and ions (protons) of equal density $n_{0}$. We define the frequency and wave number of the waves as $\omega$ and $\mathbf{k}$, respectively. If not otherwise noted, the $x$ axis is taken along the background magnetic field $\mathbf{B}_{0}$, and the $z$ axis is so chosen that the wave vector $\mathbf{k}$ is in the $x-z$ plane, $\mathbf{k} \equiv\left(k_{x}, 0, k_{z}\right)=k(\cos \theta, 0, \sin \theta)$. Linear dispersion property is summarized in Appendix A. We neglect the back reaction of electrons toward the electromagnetic field (test-particle approach).

1 Here we have chosen the electron motion. Of course, we can equally take the ion motion with a suitable modification of the choice of wave modes in the following consideration of resonant interactions. The relativistic effect will be considered in $\$ 5.4$ and $\$ 5.5$

2 Note, however, that there still remains self nonlinearity in (3) for the mode \#, where $\mathbf{E}_{w}^{\#}$ and $\mathbf{B}_{w}^{\#}$ are evaluated at the particle position $\mathbf{r}_{e}(t)$, which is affected by $\mathbf{E}_{w}^{\#}$ and $\mathbf{B}_{w}^{\#}$ themselves. This nonlinearity is the origin of subharmonic resonances to be discussed in 4.5 


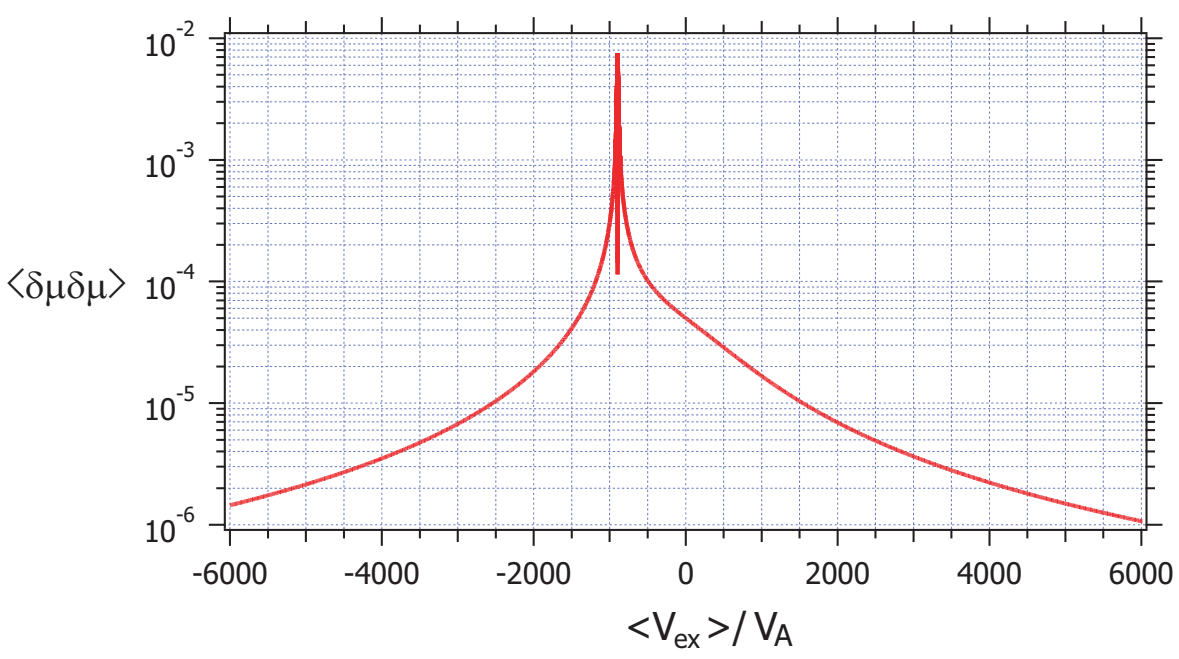

Fig. 1 The result of test-particle calculation for a parallel propagating wave with $\omega=5 \Omega_{c i}=$ $2.72 \times 10^{-3} \Omega_{c e}, k=k_{x}=2.044 \Omega_{c i} / V_{A}$, and $\left|\mathbf{B}_{w}\right| / B_{0}=10^{-4}$. The variance of $\mu$, the cosine of the pitch angle, is plotted against the average velocity $\left\langle V_{e x}\right\rangle$, where the horizontal axis is normalized by the Alfvén velocity $V_{A}$. The peak at $\left\langle V_{e x}\right\rangle / V_{A}$ at $\sim-892.2$ shows the effect of the fundamental cyclotron resonance condition.

\section{Parallel propagating waves}

The first case is for a parallel propagating whistler wave with $\omega=5 \Omega_{c i}=2.72 \times$ $10^{-3} \Omega_{c e}, k=k_{x}=2.044 \Omega_{c i} / V_{A}$, and the relative wave amplitude $\left|\mathbf{B}_{w}\right| / B_{0}=10^{-4}$. We inject $3.5 \times 10^{4}$ electrons with the initial conditions of

$$
r_{e}=(0,0,0) \text { and } \mathbf{V}_{e}=\left(V_{e x, 0}, V_{e \perp} \cos \alpha, V_{e \perp} \sin \alpha\right),
$$

where $\left(V_{e x, 0}, \alpha\right)$ are distributed uniformly in the two dimensional space, $\left(-6000 V_{A}\right.$, $\left.6000 V_{A}\right) \times(0,2 \pi)$, and $V_{e, \perp}$ is fixed at $1000 V_{A}$. We integrate (2) and (3) for a long enough time $T$, and then evaluate the averages of $V_{e x}, \mu$ (cosine of the pitch angle), and $\delta \mu \delta \mu$ (the variance of $\mu$ ),

$$
\begin{aligned}
& \left\langle V_{e x}\right\rangle=\frac{1}{T} \int_{0}^{T} V_{e x}(t) d t \\
& \langle\mu\rangle=\frac{1}{T} \int_{0}^{T} \frac{V_{e x}(t)}{\left|\mathbf{V}_{e}(t)\right|} d t \\
& \langle\delta \mu \delta \mu\rangle=\frac{1}{T} \int_{0}^{T}\left(\frac{V_{e x}(t)}{\left|\mathbf{V}_{e}(t)\right|}-\langle\mu\rangle\right)^{2} d t
\end{aligned}
$$

In Figure 1 $\langle\delta \mu \delta \mu\rangle$ is plotted against $\left\langle V_{e x}\right\rangle$, where we see a peak of $\langle\delta \mu \delta \mu\rangle$ at $\left\langle V_{e x}\right\rangle=$ $-892.2 V_{A} \equiv V_{R}$, where $V_{R}$ is the resonance velocity satisfying

$$
\omega-k_{x} V_{R}=n \Omega_{c e}
$$




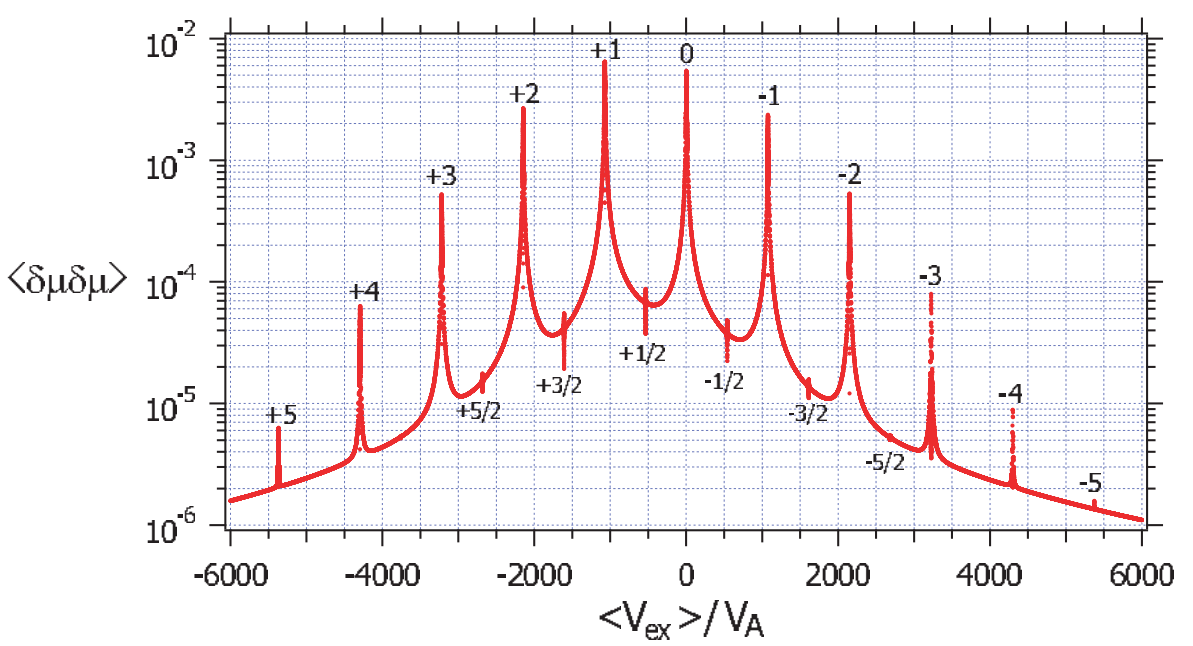

Fig. 2 The same as Figure 1 except that it is for a oblique propagating wave with $\omega=$ $5 \Omega_{c i}=2.72 \times 10^{-3} \Omega_{c e}, k=2.417 \Omega_{c i} / V_{A}, k_{x}=1.709 \Omega_{c i} / V_{A}, \theta=45^{\circ}$, and $\left|\mathbf{B}_{w}\right| / B_{0}=$ $10^{-4}$. Integers given above the peaks $(-5,-4, \ldots, 5)$ are harmonic numbers in the resonance condition, $\omega-k_{x} V_{R}=n \Omega_{c e}$. Half integers given below the curve $(-5 / 2,-3 / 2, \ldots, 5 / 2)$ are for the subharmonic resonance (see text).

with $n=+1$ (the fundamental cyclotron resonance condition). This is the most natural resonance, in which right-hand rotating electrons interact with right-hand polarized whistler waves. Historically-famous Kennel-Petscheck theory (Kennel and Petschek (1966)) for the loss-cone instability of whistler waves by the trapped electrons in the radiation belt mainly focused on this resonance. Recently, Amano and Hoshino (2010) applied this resonance condition to the electron injection process at shocks $(\$ 5.1)$.

Note that electrons in a monochromatic wave are 'trapped' around $V_{R}$ and their pitch angle diffusion does not occur. In Figure 1 there is a dip at $\left\langle V_{e x}\right\rangle=V_{R}$, which is the manifestation of the trapping effect (Appendix B). If, on the other hand, we superpose multi waves, the interaction between the electron and one particular wave component is limited within a finite coherent time $\tau$, giving a contribution $\langle\delta \mu \delta \mu\rangle / \tau$ to the pitch-angle diffusion coefficient. The task of quasilinear theory from this point of view is to evaluate the coherent time $\tau$ for a given ensemble of wave components. Since the literature on quasilinear theory is too vast, we should limit the reference to only a few articles (e.g., Kennel and Engelmann (1966); Hall and Sturrock (1967); Hasselmann and Wibberenz (1968); Kulsrud and Ferrari (1971); Jokipii (1971); Lee and Lerche (1974); Skilling (1975); Schlickeiser (1989a 1989b); Hollweg and Isenberg (2002); Schlickeiser (2002); Petrosian and Liu (2004); Shalchi (2009)).

\section{Oblique propagating waves}

Figure 2 is for an oblique propagating whistler wave with $\omega=5 \Omega_{c i}=2.72 \times 10^{-3} \Omega_{c e}$, $k=2.417 \Omega_{c i} / V_{A}, k_{x}=1.709 \Omega_{c i} / V_{A}, \theta=\pi / 4$, and the same relative wave amplitude $\left|\mathbf{B}_{w}\right| / B_{0}=10^{-4}$ as in $\$ 3$ We inject $5.0 \times 10^{4}$ electrons with the same initial condition as (4). Peaks labeled with integer numbers $(n=-5,-4, \ldots,, 5)$ correspond to the harmonic 


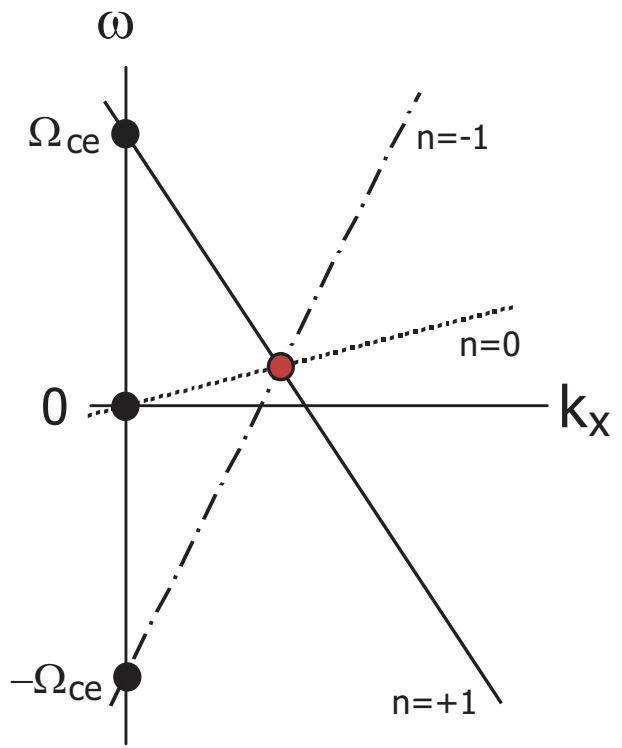

Fig. 3 Schematic illustration of the three resonance conditions, $\omega-k_{x} V_{R}=n \Omega_{c e}$, for $(n=0, \pm 1)$ for an electron with a whistler wave (red circle).

number $n$ in the resonance condition (6). It is noted that there sometimes appear dips at the resonant velocities. Again these dips are manifestation of the trapping effect already seen in Figure 1. The resonance conditions for $n=0, \pm 1$ are illustrated in Figure 3.

4.1 Fundamental cyclotron resonance with $n=+1$

The highest peak with $n=+1$ in Figure 2 is for the fundamental cyclotron resonance condition,

$$
\omega-k_{x} V_{R}=+\Omega_{c e}
$$

which is already discussed in 3 for the purely parallel propagating case.

4.2 Transit-time resonance with $n=0$

The second highest peak in Figure 2 is for the Landau resonance or transit-time resonance condition,

$$
\omega-k_{x} V_{R}=0
$$

To see the origin of this resonance, we return to the $x$ component of the equation of motion (3),

$$
\frac{d V_{e x}}{d t}=-\frac{e}{m_{e}}\left\{E_{w, x}+\frac{1}{c}\left(V_{e, y} B_{w, z}-V_{e, z} B_{w, y}\right)\right\}
$$

where the second term in the parenthesis on the right hand side of (9) is the $\left(\mathbf{V}_{e} \times \mathbf{B}_{w}\right)_{x}$ term. From the numerical inspection of (9), we find that the relative contribution of 
the $E_{w, x}$ term is less than $1 \%$ of the $\left(\mathbf{V}_{e} \times \mathbf{B}_{w}\right)_{x}$ term. (The dependence of $\langle\delta \mu \delta \mu\rangle$ on $\left\langle V_{e x}\right\rangle$ is essentially unchanged, if $E_{w, x}$ is artificially set zero.) The $(\mathbf{V} \times \mathbf{B})_{x}$ term describes the mirror force exerted by the variation of the magnetic field magnitude, and this resonance is called 'transit-time' resonance (e.g., Schlickeiser and Miller (1998)).

4.3 Anomalous cyclotron resonance with $n=-1$

The resonance corresponding to the peak with $n=-1$ in Figure 2

$$
\omega-k_{x} V_{R}=-\Omega_{c e}
$$

is historically called 'anomalous cyclotron resonance' (or 'anomalous Doppler effect': e.g., Ginzburg (1960); Brice (1964)). (10) is equivalent to

$$
\frac{\omega}{k_{x}}-V_{R}=-\frac{\Omega_{c e}}{k_{x}} \quad(<0)
$$

which means that the wave is overtaken by the electron as $V_{R}$ exceeds the wave phase speed $\omega / k_{x}$ (here we set $k_{x}>0$ ). Therefore, there is a reversal of the wave polarization from the background plasma frame to the electron comoving frame. The term 'anomalous' stems from this reversal of polarization. It is noted that an oblique propagating whistler wave is elliptically polarized and consists of right-hand polarized (R) and lefthand polarized (L) subcomponents. When the resonance condition (10) applies, there are dual polarization reversals, namely $\mathrm{R}$ and $\mathrm{L}$ subcomponents in the background plasma frame respectively become left-hand and right-hand polarized in the electron comoving frame. What the electron efficiently exchanges energy and momentum at the $n=-1$ condition is the $\mathrm{L}$ subcomponent in the background plasma fram 3 . Levinson (1992) utilized this resonance condition to excite oblique whistler waves by electron beams injected from the shock front.

\subsection{Cyclotron higher-harmonic resonance at $n$ with $|n| \geq 2$}

Cyclotron higher-harmonic resonance phenomena for oblique and perpendicular propagating waves are well known (see, e.g., Chapter 10 of Stix (1992)). Its application to Bernstein wave observed in the Jovian magnetosphere is one of the classical examples (Barbosa and Kurth (1980)).

4.5 Cyclotron sub-harmonic resonance at half integers

In Figure 2 there are some minor structures at subharmonic numbers,

$$
n=-5 / 2,-3 / 2, . ., 3 / 2,5 / 2 \text {, }
$$

where the dip structures (trapping effects) are seen to dominate over the small peak structures. These are manifestations of cyclotron sub-harmonic resonance interaction,

3 From this consideration it is naturally understood why there is no anomalous resonance effect for a parallel propagating whistler wave (Figure 1): An electron satisfying the $n=-1$ resonance condition feels the whistler wave left-hand polarized in its own comoving frame and does not exchange energy and momentum with the wave efficiently. 


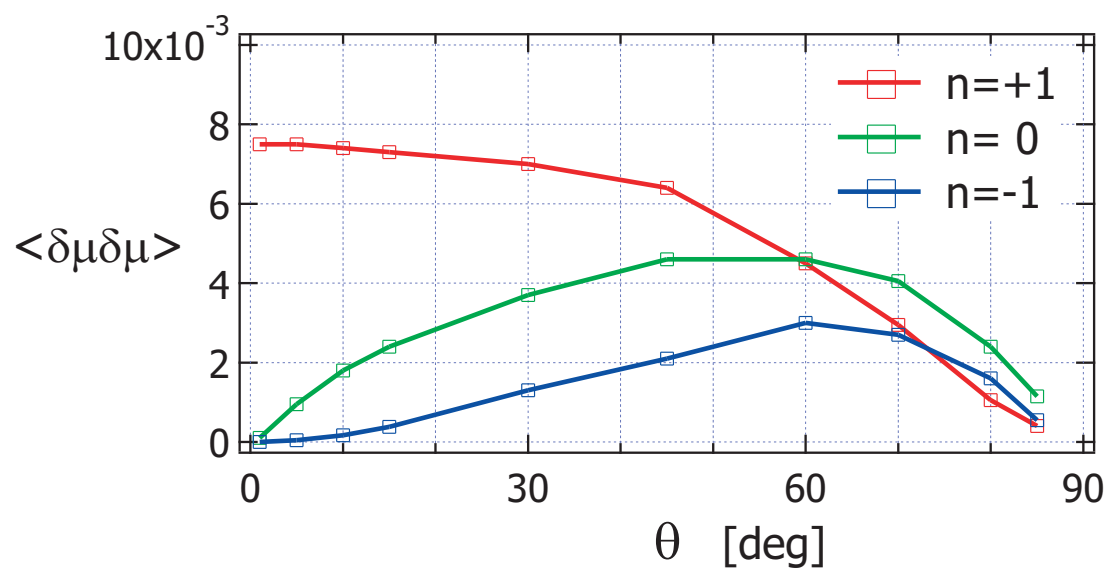

Fig. 4 Angular dependence of $\langle\delta \mu \delta \mu\rangle$ for whistler waves at three different resonance conditions $(n=0, \pm 1)$ is shown for $\theta=0^{\circ} \sim 85^{\circ}$. The wave frequencies and relative amplitudes are fixed at $\omega=5 \Omega_{c i}=2.72 \times 10^{-3} \Omega_{c e}$, and $\left|\mathbf{B}_{w}\right| / B_{0}=10^{-4}$, respectively.

which was first discussed by Smirnov and Frank-Kamenetskii (1968), and then followed by Terasawa and Nambu (1989) for perpendicular propagating magnetosonic waves. There are renewed interests on this particular resonance for oblique Alfvén waves (e.g., Chen et al. (2001); White, Chen and Lin (2002); Lu and Chen (2009)). The origin of this resonance is the self nonlinearity noted in the footnote 2 of 92

4.6 Angular dependence of $\langle\delta \mu \delta \mu\rangle$

As shown in Figure 2, there are many possible resonance modes for oblique propagating waves. It is of interest to see how the relative importance of these mode depends on the propagation angle $\theta$. Figure 4 shows the dependence of $\langle\delta \mu \delta \mu\rangle$ on the propagation angle $\theta$ of whistler waves at three different resonance conditions $(n=0, \pm 1)$. In the quasi-parallel propagation regime $\left(\theta<\sim 45^{\circ}\right)$, the fundamental resonance of $n=+1$ dominates over others. On the other hand, in the quasi-perpendicular regime $(\theta>\sim$ $\left.45^{\circ}\right)$, the transit-time resonance $(n=0)$ and the anomalous cyclotron resonance $(n=$ -1 ) become progressively more important as $\theta \rightarrow 90^{\circ}$, and exceed the fundamental resonance above $\theta \sim 60^{\circ}-70^{\circ}$. 


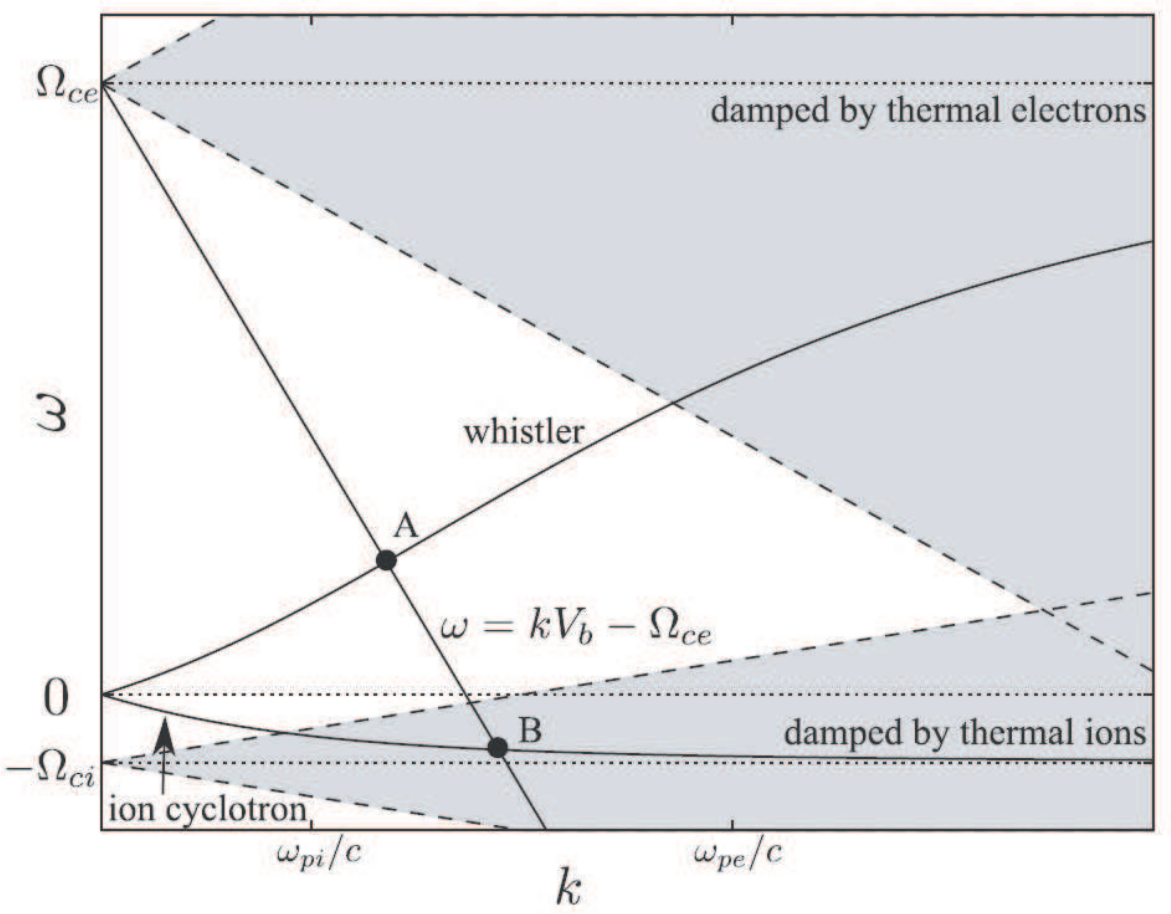

Fig. 5 From Amano and Hoshino (2010). Schematic dispersion diagram for whistler and ioncyclotron waves in an electron-ion plasma. Positive (negative) frequency corresponds to the right-hand (left-hand) polarization. The cyclotron resonance condition (7) is also shown. Waves in the shaded regions are strongly damped by the cyclotron damping of thermal plasma particles (electrons and ions).

\section{Recent topics of resonant particle interactions}

5.1 A critical Mach number for electron injection in shocks

It has been known that the electron component of galactic cosmic rays is about $1 \%$ of the proton component (e.g., Schlickeiser (2002)). While the origin of this percentage is not well understood, it is generally interpreted as the indication of smaller injection/acceleration efficiency for electrons than ions. For the case of ions, a part of their suprathermal component is reflected by shock fronts to form a beam. The beam ions then excite MHD waves through the ion-beam-cyclotron resonance condition and are subject to the pitch angle scattering process (Winske and Leroy (1984)), which is the first step to the diffusive shock acceleration process for the ions. If we apply the same scenario to electrons, difficulty arises at the level of the wave excitation. Figure 5 illustrate this difficulty (Amano and Hoshino (2010)): An oblique solid line running from the upper-left is the fundamental cyclotron resonance condition (77) for the electrons with the beam velocity, $V_{\mathrm{e}, \text { beam }}$,

$$
\omega-k_{x} V_{\mathrm{e}, \text { beam }}=\Omega_{c e}
$$



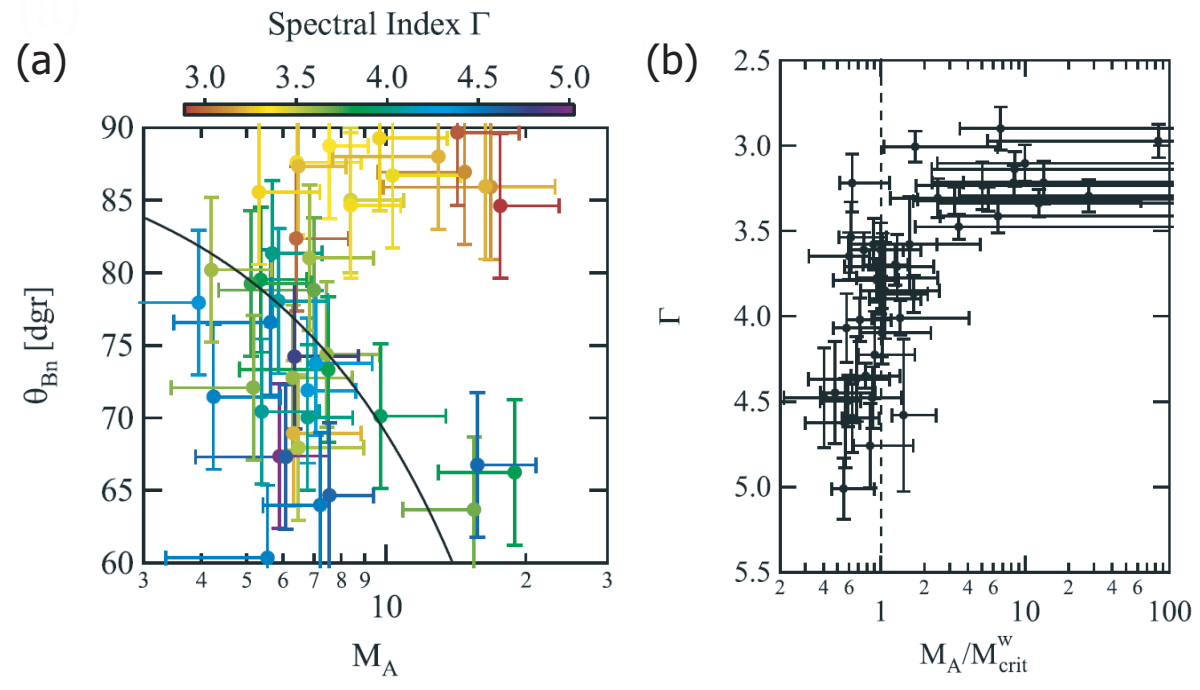

Fig. 6 From Oka et al. (2006). (a) Observed spectral indices $\Gamma$ of suprathermal electrons at the bow shock transition region are plotted onto the two dimensional map of observed parameters $\left(M_{A}, \theta_{B n}\right) . \Gamma$ is color-coded from orange (hard) to dark blue (soft). A solid black curve shows the position of the critical Mach number $M_{A \text {, crit }}$ where $\beta$ is set 1 . (b) Observed spectral indices $\Gamma$ are plotted against the Mach number normalized by $M_{A, \text { crit }}$ where observed $\theta_{B n}$ is used to calculate $M_{A, c r i t}$.

The interaction point ' $\mathrm{A}$ ' between the beam electrons and the whistler wave is not available for the wave excitation since the beam electrons with purely parallel velocity $a b s o r b$ whistler wave energy at this point. The interaction point ' $\mathrm{B}$ ' is for the anomalous cyclotron resonance condition between the beam electrons and ion cyclotron wave (left-hand polarized in the plasma rest frame). However, in the interplanetary and interstellar plasmas of typical temperature, there are a plenty of thermal ions which damp the ion cyclotron wave via fundamental cyclotron resonance interaction (shaded region around 'B') so that efficient pitch angle scattering of electrons is prohibited.

What Amano and Hoshino (2010) proposed is to invoke the loss cone distribution of beam electrons, which is the natural consequence of the electron reflection process at the shock front (shock drift acceleration process) and can provide the free energy to the whistler wave excitation at the interaction point 'A' if the damping effect by thermal electrons (shaded region around the $\omega=\Omega_{c e}$ line, namely $-k_{x} v_{e, t h}+\Omega_{c e}<$ $\omega<k_{x} v_{e, t h}+\Omega_{c e}$ with the thermal velocity $v_{e, t h}$ of background electrons) is avoided. These considerations lead to the criterion for the shock Alfvén Mach number $M_{A}$ for the efficient electron acceleration,

$$
M_{A} \geq M_{A, \text { crit }} \equiv \frac{\cos \theta_{B n}}{2} \sqrt{\frac{m_{i}}{m_{e}} \beta_{e}}
$$

which agrees with the observational criterion for electron acceleration obtained at the earth's bow shock, where the hard spectral indices of suprathermal electrons $(\Gamma \leq 3.5)$ are observed preferentially for high Mach number bow shock satisfying $M_{A}>M_{A, \text { crit }}$ (Figure 6 from Oka et al. (2006)). 


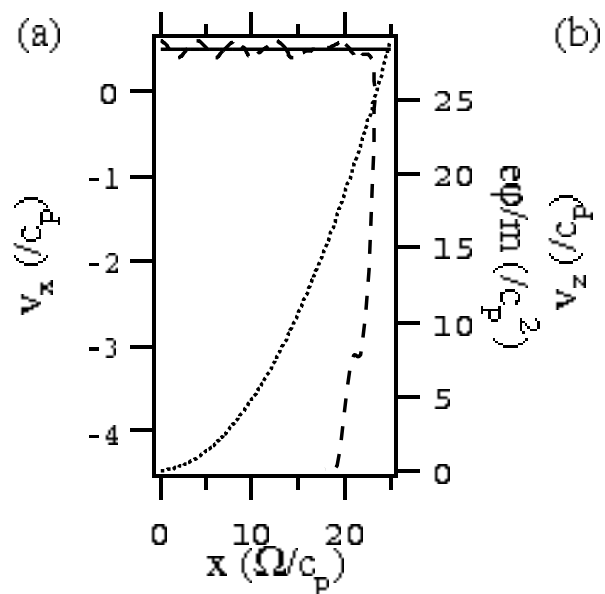

(b)
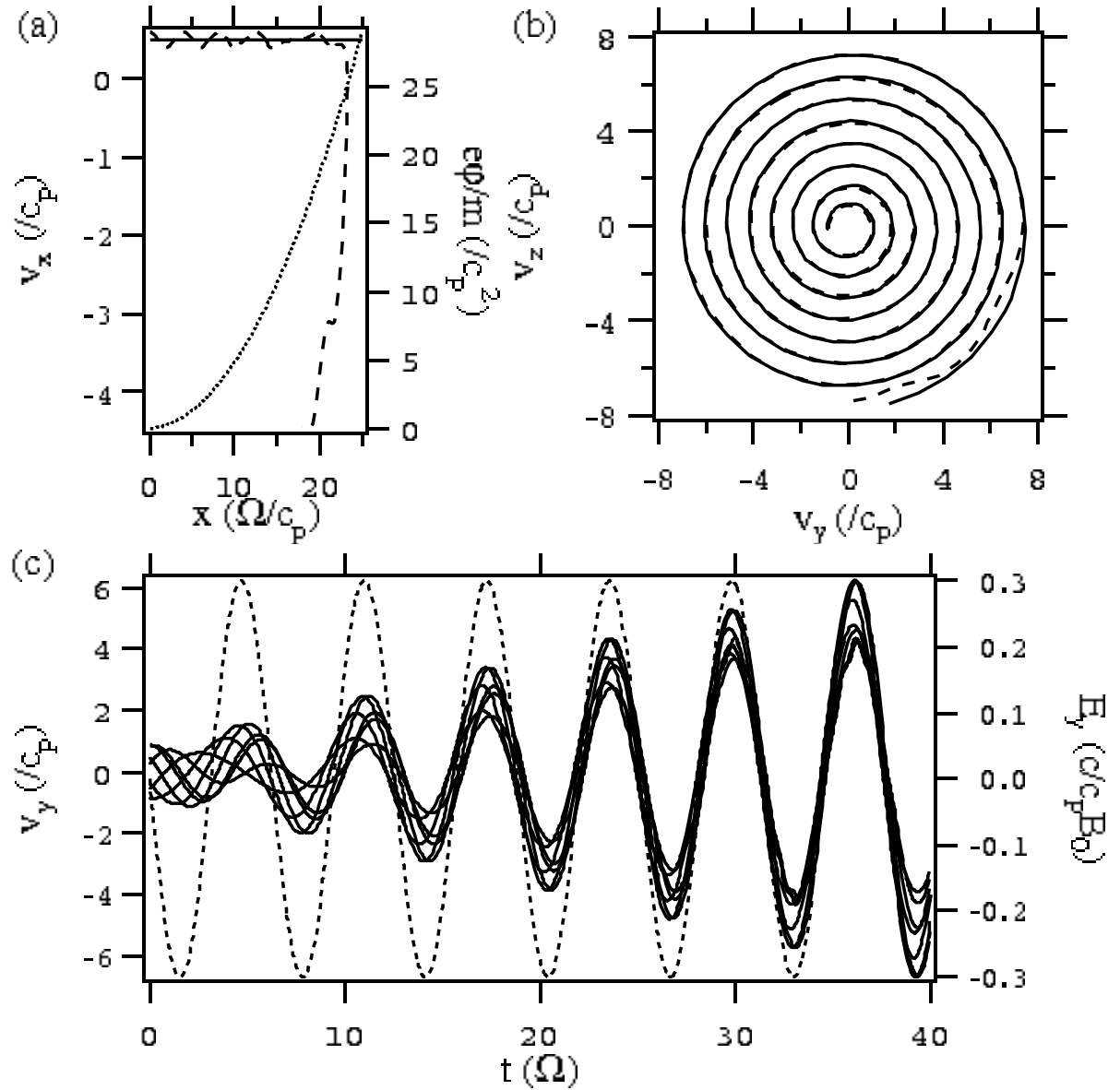

Fig. 7 Figures from Kuramitsu and Krasnoselskikh (2005a). Trajectories of ions satisfying a forced resonance condition with an electromagnetic wave under an external electrostatic field. Resonant (solid lines) and near-resonant (dashed lines) ions in (a) $v_{x}-x$ and (b) $v_{z}-v_{y}$ phase spaces. A dotted line in (a) denotes the spatial profile of the electrostatic field. (c) Time evolution of $v_{y}$ of the resonant ions (solid lines) and $E_{y}$ (dotted line).

\subsection{Gyroresonant surfing acceleration}

Kuramitsu and Krasnoselskikh (2005a) proposed a new type of surfing acceleration of ions resonating with a left-hand circularly polarized wave through the fundamental cyclotron resonance condition 4 with $n=+1$,

$$
\omega-k_{x} V_{R}=\Omega_{c i}
$$

It is known that a particle trapped by a monochromatic circularly polarized wave oscillates around the resonant point in a phase space. During a trapped oscillation, a

\footnotetext{
4 In this and next subsections we consider the resonant interaction of ions and set the sign of $\omega$ positive for the left-hand polarized waves.
} 
(a)
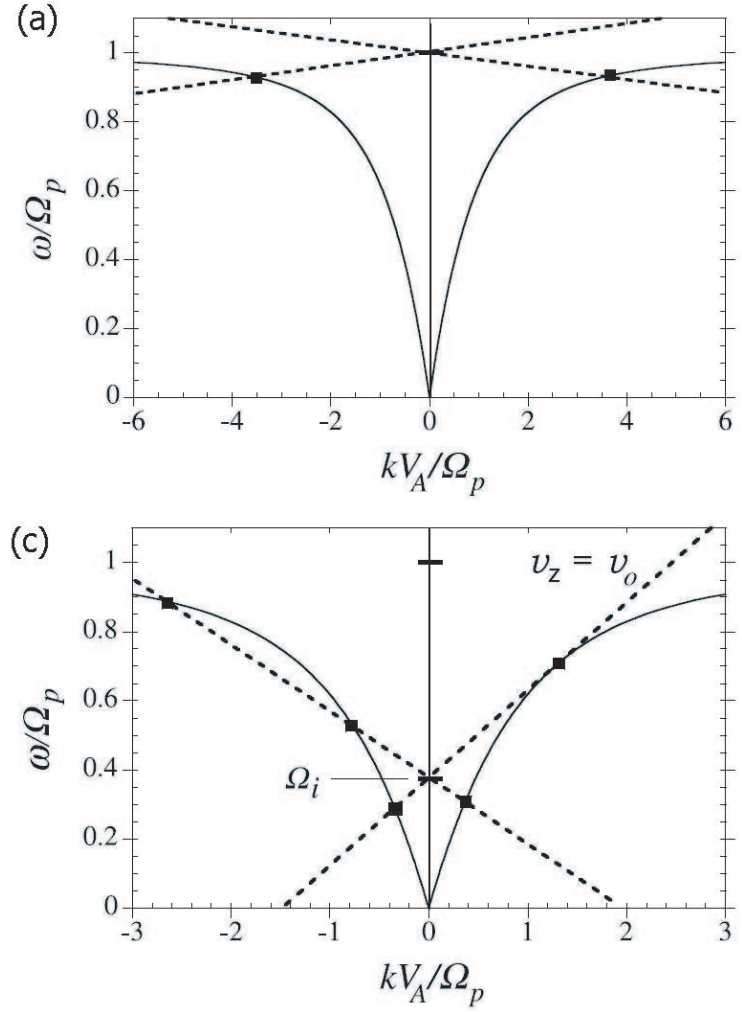
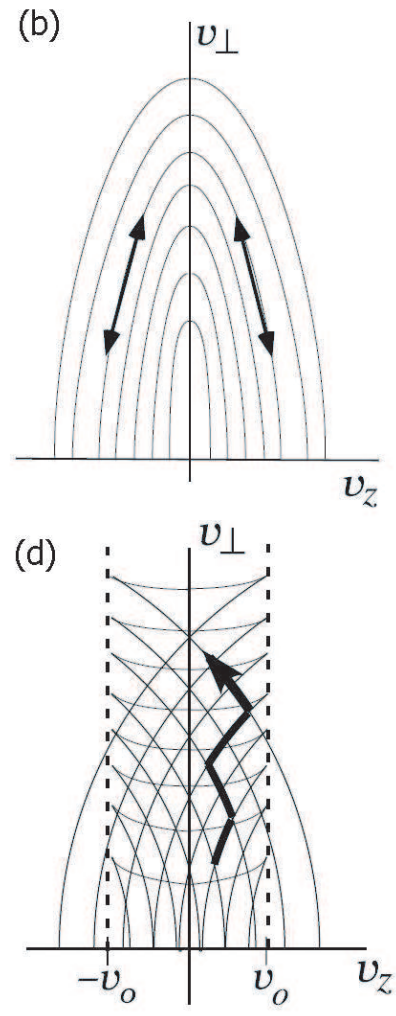

Fig. 8 From Isenberg and Vasquez (2007). (a) The cyclotron resonance condition between protons and parallel-propagating ion cyclotron waves. The solid lines plot the wave dispersion relation, while the dashed lines show the resonance condition for two particular proton parallel velocities with solid squares indicating the resonance points with the waves. (b) Resonant surfaces for protons in velocity space, $\left(v_{z}-\omega / k\right)^{2}+v_{\perp}^{2}=$ const. The cyclotron interaction causes protons to diffuse along these surfaces, but not across them. (c) and (d) Similar to (a) and (b) but for a minor ion species with $\Omega_{i}<\Omega_{p}$.

particle energy as well as a relative phase between the particle and the wave is bounded. However, if an external force is imposed to keep the relative phase constant, the particle can continue to gain energy from the wave electric field.

Such a forced resonance condition is realized, for instance, in the foreshock region of a quasi-parallel shock where an electrostatic potential acts as the external force. Figure 7 shows completely resonant (solid lines) and near resonant (dashed lines) ion trajectories in $v_{x}-x(\mathrm{a})$ and $v_{z}-v_{y}$ (b) phase spaces in a given electrostatic potential whose spatial profile is represented with a dotted line in (a). The two particles are accelerated monotonically in perpendicular direction while keeping the parallel velocities (almost) constant at $v_{x} \approx V_{R}(=0.5)$. In their test particle simulation all the resonant particles are eventually accelerated regardless of their initial gyro phases due to the effect of phase synchronization which is shown in Figure 7 (c) in which time evolution of $v_{y}$ of the resonant particles having a variety of initial gyro phases (solid lines) and $E_{y}$ component of the wave electric field (dotted line) are plotted. 

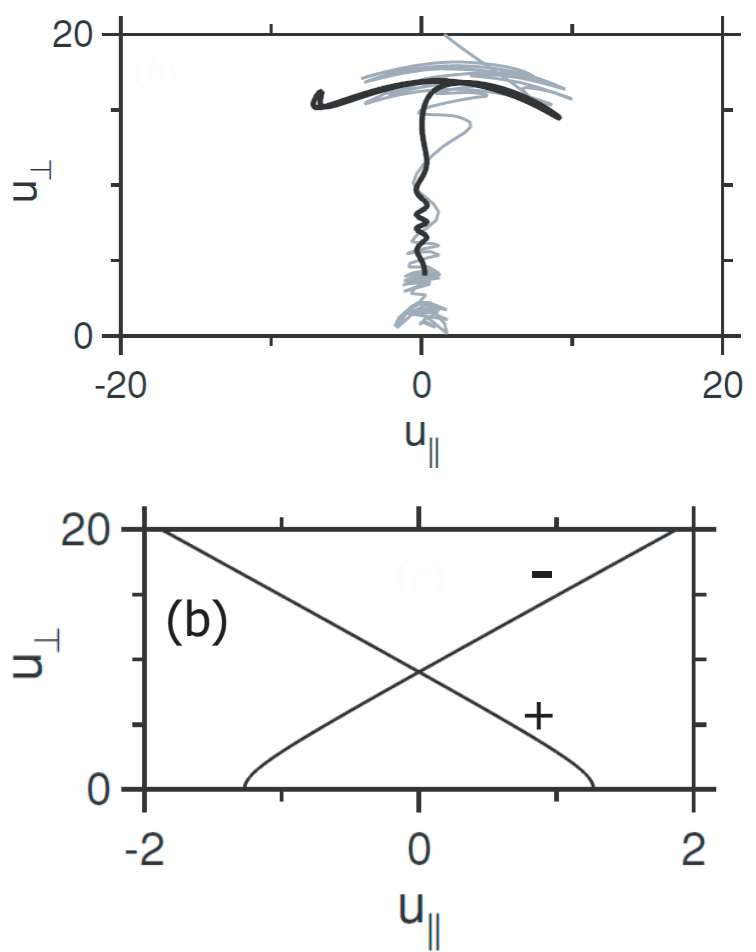

Fig. 9 Figures from Matsukivo and Hada (2009). (a) Two four-velocity trajectories (black and gray curves) of relativistic electrons which are efficiently accelerated. (b) Relativistic fundamental cyclotron resonance conditions (16) for positive $(+)$ and negative $(-)$ parallel wave numbers.

\subsection{Cyclotron resonant heating of solar wind heavy ions}

It has been known that heavy ions in the fast solar wind have faster flow velocity and hotter temperature (especially in the direction perpendicular to $\mathbf{B}_{0}$ ) than the proton population, the main population of the solar wind. Isenberg and Vasquez (2007) have proposed a second-order Fermi acceleration mechanism for the preferential perpendicular heating of heavy ions by counterstreaming left-hand circularly polarized waves. In Figure 8, the case for protons ((a) and (b)) is contrasted with that for heavy ions $((\mathrm{c})$ and $(\mathrm{d}))$ : The second-order Fermi process works rather slowly for protons. This is because the signs of the resonance velocities for parallel and anti-parallel propagating waves are different, and the acceleration of protons by these waves does not occur simultaneously. On the other hand, minor heavy ions whose cyclotron frequency $\Omega_{i}$ is smaller than $\Omega_{p}$ have multiple resonant interaction points if the condition $\left|v_{z}\right|<v_{0}$ is satisfied (Figure 8 (c) $\sqrt[5]{5}$. Therefore, heavy ions can be accelerated by multi waves simultaneously as indicated by the thick arrow in Figure 8(d).

5 The authors took the $z$ axis along $\mathbf{B}_{0}$. The limiting velocity $v_{0}$ for the multiple interactions corresponds to the condition tangential to the wave dispersion relation (Figure 8] (c)). 
5.4 Relativistic particle acceleration in developing turbulence

Matsukivo and Hada (2009) found a very efficient acceleration of relativistic particles in developing Alfvén waves in an electron-positron pair plasma, where the wave number and frequency spectra of the waves are developing through parametric decay instabilities. Figure 9 (a) shows two examples of trajectories of electrons which are efficiently accelerated along the vertical line $\left(u_{\|}=0\right)$. The gray line denotes an accelerated electron in the course of the decay instability self-consistently reproduced in a onedimensional full particle-in-cell simulation, while the black line is obtained from a test particle simulation in modeled electromagnetic fields. This acceleration is interpreted in the following scenario: In the developing turbulence, there exist waves propagating both directions parallel and anti-parallel to the background magnetic field. The fundamental cyclotron resonance condition $(n=+1)$ for relativistic electrons is $V_{x}=V_{R}$ where $V_{R}$ satisfies

$$
\omega-k_{x} V_{R}=\frac{\Omega_{c e}}{\gamma}
$$

With the four velocity $\left(u_{\|}, u_{\perp}\right) \equiv\left(v_{x} \gamma / c, v_{\perp} \gamma / c\right)$, (15) is rewritten a: 6 ,

$$
\left(\frac{\omega}{\Omega_{c e}}\right)\left(1+u_{\|}^{2}+u_{\perp}^{2}\right)^{1 / 2}=1+\left(\frac{k_{x} c}{\Omega_{c e}}\right) u_{\|}
$$

Figure 9 (b) shows the relation between $u_{\|}$and $u_{\perp}$ given by (16), where the frequency $\omega / \Omega_{c e}=0.11$ and the parallel and anti-parallel wave numbers $k_{x} c / \Omega_{c e}= \pm 0.65$ are chosen. It is remarkable that at $u_{\|}=0$ the two resonance curves overlap so that an electron can interact with two counterstreaming waves at the same time and be efficiently accelerated. This scenario is similar to the one for heavy ions (\$5.3) except that the former is made possible by the relativistic reduction of the cyclotron frequency $\left(\Omega_{c e} / \gamma<\Omega_{c e}\right)$ instead of the intrinsic difference in cyclotron frequencies for the latter $\left(\Omega_{i}<\Omega_{p}\right)$.

\subsection{Relativistic turning acceleration}

Recently, it is suggested that relativistic electrons in the earth's radiation belt are coherently accelerated by large amplitude whistler waves satisfying the relativistic cyclotron resonance condition (15) (e.g., see Omura et al. (2007); Furuya et al. (2008); Tao and Bortnik (2010)). Large amplitude whistler waves are actually observed as 'chorus' emissions (Cattell et al. (2008); Cully et al. (2008)). The essence of the 'turning acceleration' model (Omura et al. (2007); Furuya et al. (2008)) is the relativistic reduction of the cyclotron frequency in (15). Figure 10 shows two cases with $V_{R}<0$ (with $\gamma=\gamma_{E}$ ) and $V_{R}>0$ (with $\gamma=\gamma_{D}>\gamma_{E}$ ). An electron with $\gamma_{E}$ and $V_{R}<0$ is assumed to start interacting with the (counter-streaming) chorus wave. Since the chorus wave has a large amplitude, the electron is trapped and accelerated to increase $\gamma$ (i.e., reduce $V_{R}$ ) coming to the turning point with $V_{R}=0$. After the turning point, the electron moves toward the same direction of the wave propagation, so that the

6 Note the following relation for the Lorentz factor,

$$
\gamma \equiv\left\{1-\left(v_{x}^{2}+v_{\perp}^{2}\right) / c^{2}\right\}^{-1 / 2}=\left(1+u_{\|}^{2}+u_{\perp}^{2}\right)^{1 / 2} .
$$


(a)

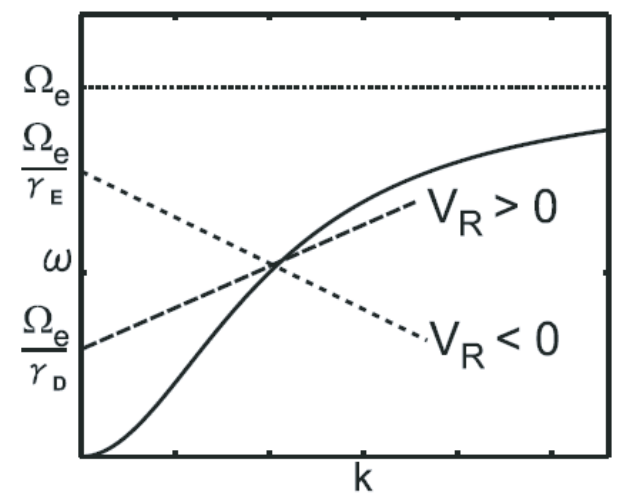

(b)

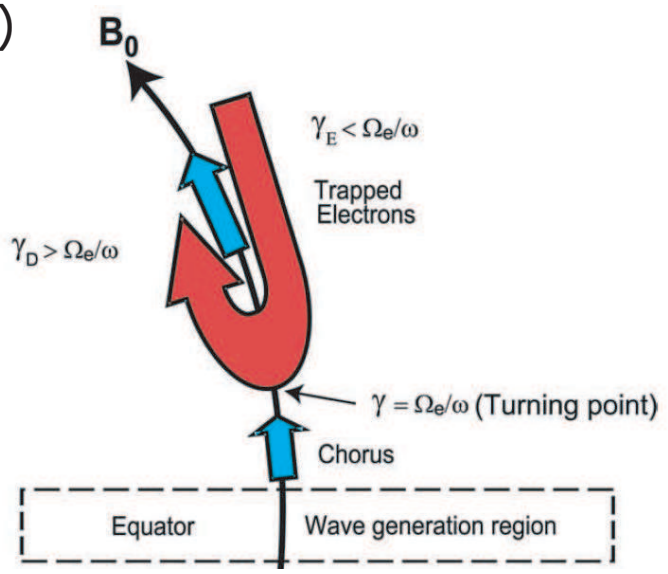

Fig. 10 Figures from Omura et al. (2007). (a) Fundamental relativistic cyclotron condition (15). (b) Illustration of 'relativistic turning acceleration'. Large amplitude chorus waves are assumed to be excited near the equator and propagate from there to the high latitude region along the field line. An electron coming from the low altitude mirror point toward the equator starts interacting with the chorus wave.

wave-particle interaction to continue long enough to further accelerate the electron up to $\gamma=\gamma_{D}$, where the electron is detrapped.

\section{Concluding remarks}

We have given an overview on the importance of resonant wave-particle interaction processes in space plasmas. Although each of elementary processes are well known one by one, there is a necessity to unify these processes. For example, as we see in 44 to consider interaction process between particles and oblique propagating waves we need to take into account of all resonance interactions with harmonic numbers $n=0, \pm 1, \pm 2, \ldots$, and sometimes even with subharmonic numbers $n= \pm 1 / 2, \pm 3 / 2, \ldots$. 
In $\S 5.1-3$ we see the various roles of cyclotron resonant condition in the processes, shock injection, gyroresonant surfing, and ion heating in the solar wind. Of course, the considerations for the elementary resonance processes alone do not suffice for comprehensive understandings of targeting physical processes: Injected electrons at the shock front (\$5.1) or surfing-accelerated ions at the shock front (\$5.2) should be accelerated further in diffusive shock acceleration process, for example, to acquire relativistic energy. For the dynamics of heavy ions in the solar wind, the second-order Fermi heating mechanism (\$5.3) is incorporated into an inhomogeneous coronal hole model with the effects of gravity, charge-separation electric field, and mirroring force in the decreasing solar magnetic field (Isenberg and Vasquez (2009)).

In $\$ 5.4$ and $\$ 5.5$ We further see that the relativistic resonance condition makes the interaction process very rich. Note that these considerations are for the behavior of relativistic particles in nonrelativistic turbulences. While there are a few pioneering studies in stochastic acceleration process in relativistic turbulences (e.g., Viratanen and Vainio, (2005); O'Sullivan et al. (2009)), further works on relativistic turbulence are needed to get comprehensive understanding, for example, of the acceleration process of ultrahigh-energy cosmic rays (e.g., Hillas (1984)).

Since we have limited the discussion to the waves in cold plasmas, wave modes raising from the finite temperature effect are outside of our scope. Among these modes, kinetic Alfvén waves have been a focus of extensive investigation. Here we refer the interested readers to a few representative papers (e.g., Hasegawa and Chen (1976); Leamon et al. (1999); Lvsak and Lotko (1996)).

Acknowledgements We would like to thank for valuable discussions and comments to $\mathrm{T}$. Hada, M. Scholer, M. A. Lee, and Y. Ohira. The works by T.T. and S. M. get partial supports from the grants-in-aids 21540259 and 22740323 from Japan Society for the Promotion of Science, respectively.

\section{Appendix A}

For the $j$ species $\left(j=i\right.$ or $e$ ), the plasma frequency is defined as $\omega_{p j}=\sqrt{4 \pi n_{0} e^{2} / m_{j}}$, and the cyclotron frequency as $\Omega_{c j}=e B_{0} / m_{j} c$. The Alfvén velocity $V_{A}$ is defined as $B_{0} / \sqrt{4 \pi n_{0}\left(m_{i}+m_{e}\right)}$, which is chosen to be $10^{-4} c$ in the test particle calculation. The ion mass $m_{i}$ is taken 1836 times $m_{e}$.

The wave electric field $\mathbf{E}_{w} \propto \exp i(\mathbf{k r}-\omega t)$ should satisfy the following matrix relation (see, e.g., Chapter 2 of $\underline{\operatorname{Stix}}(\underline{1992}))$,

$$
\tilde{M} \mathbf{E}_{w}=0
$$

with a matrix $\tilde{M}$ given as

$$
\tilde{M} \equiv\left\{\begin{array}{ccc}
P-N^{2} \sin ^{2} \theta & 0 & N^{2} \sin \theta \cos \theta \\
0 & S-N^{2} & -i D \\
N^{2} \sin \theta \cos \theta & i D & S-N^{2} \cos ^{2} \theta
\end{array}\right\}
$$


where $N \equiv k c / \omega$ is a refractive index, and $P, S$, and $D$ are defined as

$$
\begin{gathered}
P=P(\omega) \equiv 1-\frac{\omega_{p i}^{2}}{\omega^{2}}-\frac{\omega_{p e}^{2}}{\omega^{2}} \\
S=\frac{1}{2}(R(\omega)+L(\omega)) \\
D=\frac{1}{2}(R(\omega)-L(\omega)) \\
R(\omega) \equiv 1-\frac{\omega_{p i}^{2}}{\omega\left(\omega+\Omega_{c i}\right)}-\frac{\omega_{p e}^{2}}{\omega\left(\omega-\Omega_{c e}\right)} \\
L(\omega) \equiv 1-\frac{\omega_{p i}^{2}}{\omega\left(\omega-\Omega_{c i}\right)}-\frac{\omega_{p e}^{2}}{\omega\left(\omega+\Omega_{c e}\right)}
\end{gathered}
$$

Note that the condition $\operatorname{det} \tilde{M}=0$ gives the wave dispersion relation.

From the first and second column of the matrix equation (17), we have

$$
\begin{gathered}
E_{w, x}=-\frac{N^{2} \sin \theta \cos \theta}{P-N^{2} \sin ^{2} \theta} E_{w, z} \\
E_{w, y}=\frac{i D}{S-N^{2}} E_{w, z}
\end{gathered}
$$

Now switching from the complex formulation to the real formulation, we write the $x y z$ components of the electric field, $\left(E_{w, x}, E_{w, y}, E_{w, z}\right)$, as

$$
\left(E_{w, x}, E_{w, y}, E_{w, z}\right)=\left(g_{x} \cos \varphi, g_{y} \sin \varphi, g_{z} \cos \varphi\right)
$$

where the relative amplitudes of $\left(g_{x}, g_{y}, g_{z}\right)$ are determined by (20), and $\varphi$ is the phase angle,

$$
\varphi \equiv \mathbf{k} \cdot \mathbf{r}-\omega t+\varphi_{0}
$$

where $\varphi_{0}$ is the initial phase angle, respectively. From the Faraday's law, we have

$$
\mathbf{B}_{w}=N(\cos \theta, 0, \sin \theta) \times \mathbf{E}_{w}
$$

from which the $x y z$ components of the magnetic field, $\left(B_{w, x}, B_{w, y}, B_{w, z}\right)$, are obtained as

$$
\begin{aligned}
& B_{w, x}=-N \sin \theta E_{w, y} \quad=-N g_{y} \sin \theta \sin \varphi, \\
& B_{w, y}=N\left(\sin \theta E_{w, x}-\cos \theta E_{w, z}\right)=N\left(g_{x} \sin \theta-g_{z} \cos \theta\right) \cos \varphi, \\
& B_{w, z}=N \cos \theta E_{w, y} \quad=\quad N g_{y} \cos \theta \sin \varphi
\end{aligned}
$$




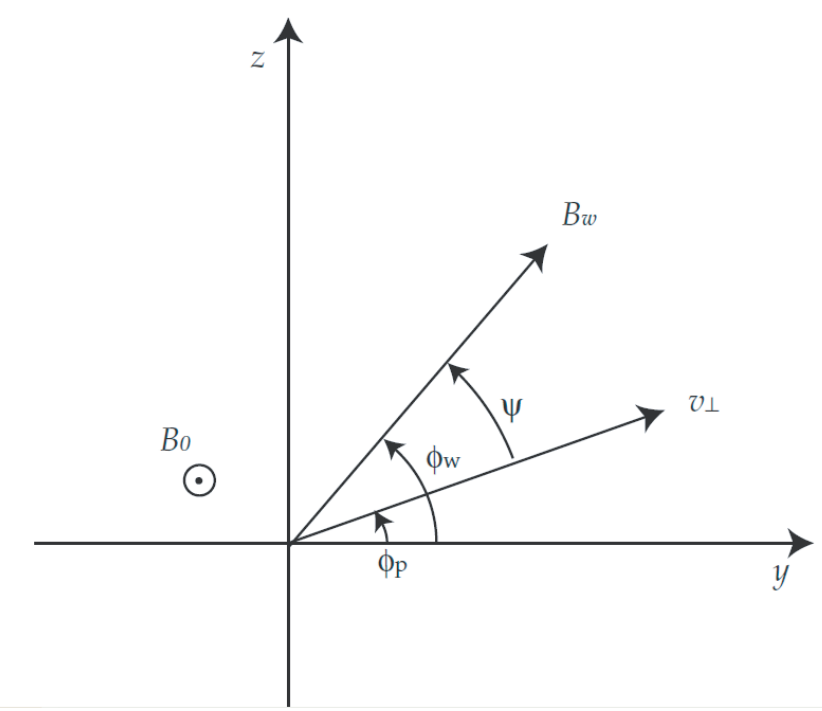

Fig. 11 Figure from Kuramitsu and Krasnoselskikh (2005b) showing the definition of angles, $\phi_{p}, \phi_{w}$, and $\psi$.

\section{Appendix B}

In a finite amplitude monochromatic electromagnetic wave propagating parallel to the background magnetic field $\mathbf{B}_{0}$ charged particles can be phase-space trapped around the resonant velocity (see e.g., Palmadesso and Schmidt (1971); Helliwell (1974); Schmitt (1976): Karpman (1974); Matsumoto (1979); Hoshino and Terasawa (1985); Kuramitsu and Krasnoselskikh $(2005 \mathrm{~b}))$. Here we follow the description in a recent article by Kuramitsu and Krasnoselskikh (2005b). Firstly, the gyrophase angle for an ion is defined as $\phi_{p} \equiv \tan ^{-1}\left(v_{z} / v_{y}\right)$, the wave phase angle as $\phi_{w} \equiv k x-\omega t+\alpha_{w}$ ( $\alpha_{w}$ : the initial value), and their difference as $\psi \equiv \phi_{w}-\phi_{p}$ (Figure 11). Next, with the velocity of ions in the wave rest frame $\left(u_{x}, u_{\perp}\right)=\left(v_{x}-\omega / k, v_{\perp}\right)$ the cosine of the pitch angle is defined as $\mu \equiv u_{x} /|u|$, where $|u|=\left(u_{x}^{2}+u_{\perp}^{2}\right)^{1 / 2}$ is the constant of motion (namely, the ion energy is conserved in the wave rest frame). The second integral of the ion motion $\chi$ can be written as,

$$
\chi=\frac{\kappa}{2}\left(\mu+\frac{1}{\kappa}\right)^{2}+b\left(1-\mu^{2}\right)^{1 / 2} \cos \psi
$$

where $\kappa=k u / \Omega_{c i}$, and $b$ the wave amplitude normalized by $\left|\mathbf{B}_{0}\right|$. With $\chi$, the equation of motion is simplified as

$$
\dot{\mu}=-\frac{\partial \chi}{\partial \psi}, \quad \dot{\psi}=\frac{\partial \chi}{\partial \mu}
$$

Figure 12 shows the ion trajectories in the $\mu-\psi$ phase space with $\kappa=3$ and $b=0.01$, $0.1,1,10$. When the wave amplitude is small $((\mathrm{a}): b=0.01)$, ions are trapped around $(\mu, \psi)=(-1 / \kappa, \pi)$, which is due to the linear cyclotron resonant interaction. As the wave amplitude $b$ becomes larger ((c) and (d)), the trapping region also becomes larger and a new trapping region is born in a location far from the original point $(-1 / \kappa, \pi)$. 

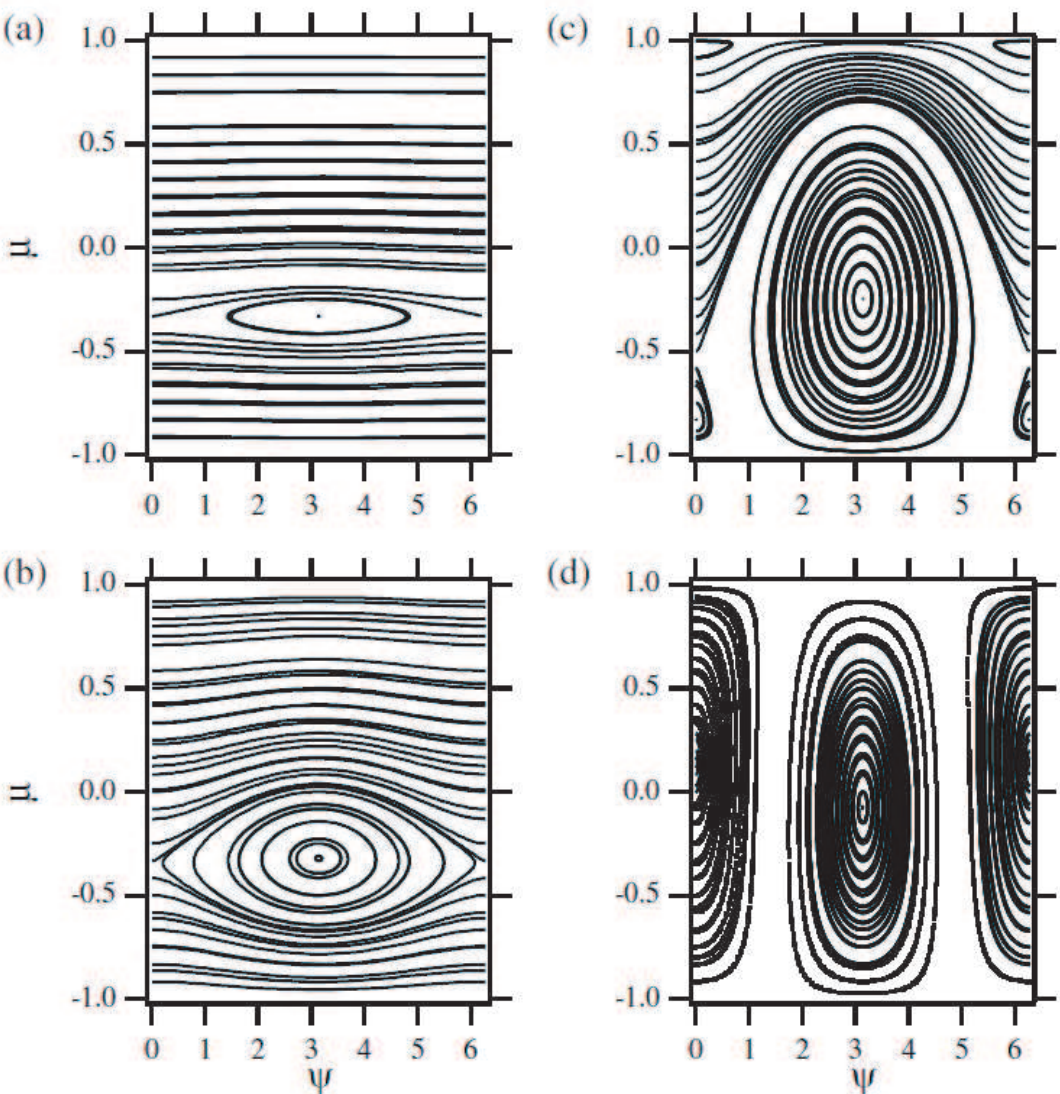

Fig. 12 Figure from Kuramitsu and Krasnoselskikh (2005b) showing particle trajectories in the $\mu-\psi$ phase space with $\kappa=3$ when (a) $b=0.01$, (b) $b=0.1$, (c) $b=1$, and (d) $b=10$. One curve corresponds to one constant of motion $\chi$.

\section{References}

Amano, T., and M. Hoshino, A critical Mach number for electron injection in collisionless shocks. Phys. Rev. Lett. 104, 181102 (2010).

Barbosa, D. D., and W. S. Kurth, Suprathermal electrons and Bernstein waves in Jupiter's inner magnetosphere. J. Geophys. Res. 85, 6729 (1980).

Brice, N., Fundamentals of very low frequency emission generation mechanisms. J. Geophys. Res. 69, 4515 (1964).

Cattell, C., J. R. Wygant, K. Goetz, et al., Discovery of very large amplitude whistler-mode waves in Earth's radiation belt. Geophys. Res. Lett. 35, L01105 (2008).

Chen, L., Z. Lin, and R. White, On resonant heating below the cyclotron frequency. Phys. Plasmas 8, 4713 (2001).

Cully, C. M., J. W. Bonnell, and R. E. Ergun, THEMIS observations of long-lived regions of large-amplitude whistler waves in the inner magnetosphere. Geophys. Res. Lett. 35, L17S16 (2008).

Fairfield, D. H., Bow shock associated waves observed in the far upstream interplanetary medium, J. Geophys. Res. 74, 3541 (1969). 
Furuya, N., Y. Omura, and D. Summers, Relativistic turning acceleration of radiation belt electrons by whistler mode chorus. J. Geophys. Res. 113, A04224 (2008).

Ginzburg, V. L., Certain theoretical aspects of radiation due to superluminal motion in a medium. Sov. Phys. Usp. 2, 874 (1960).

Hasselmann, K., and G. Wibberenz, Scattering of charged particles by random electromagnetic fields. Zeitschr. Geophys. 34, 353 (1968).

Hall, D. E., P. A. Sturrock, Diffusion, scattering, and acceleration of particles by stochastic electromagnetic field. Phys. Fluids 10, 2620 (1967).

Hasegawa, A., and L. Chen, Kinetic processes in plasma-heating by resonant mode conversion of Alfvén wave. Phys. Fluids 19, 1924 (1976).

Helliwell, R. A., Controlled VLF wave injection experiments in the magnetosphere. Space Sci. Rev. 15, 781 (1974).

Hillas, M., The origin of ultra-high-energy cosmic rays. Ann. Rev. Astron. Astrophys. 22, 425 (1984).

Hollweg, J. V., and P. A. Isenberg, Generation of fast solar wind: A review with emphasis on the resonant cyclotron interaction. J. Geophys. Res. 107, 1147 (2002).

Hoshino, M, and T. Terasawa, Numerical study of the upstream wave excitation mechanism. 1. Nonlinear phase bunching of beam ions. J. Geophys. Res. 90, 57 (1985).

Isenberg, P. A., and B. J. Vasquez, Preferential perpendicular heating of coronal hole minor ions by the Fermi mechanism. Astrophys. J. 668, 546 (2007).

Isenberg, P. A., and B. J. Vasquez, Preferential acceleration and perpendicular heating of minor ions in a collisionless coronal hole. Astrophys. J. 696, 591 (2009).

Jokipii, J. R., Propagation of cosmic rays in the solar wind. Rev. Geophys. Space Sci. 9, 27 (1971).

Karpman, V. I., Nonlinear effects in the ELF waves propagating along the magnetic field in the magnetosphere. Space Sci. Rev. 16, 361 (1974).

Kennel, C. F., and F. Engelmann, Velocity space diffusion from weak plasma turbulence in a magnetic field. Phys. Fluids 9, 2377 (1966).

Kennel, C. F., and H. E. Petschek, Limit on stably trapped particle fluxes, J. Geophys. Res. 71, 1 (1966).

Kuramitsu, Y., and V. Krasnoselskikh, Gyroresonant surfing acceleration, Phys. Rev. Lett. 94, $031102(2005 \mathrm{a})$.

Kuramitsu, Y., and V. Krasnoselskikh, Acceleration of charged particles by gyroresonant surfing at quasi-parallel shocks, Astron. Astrophys. 438, 391 (2005b).

Kulsrud, R. M., and A. Ferrari, The relativistic quasilinear theory of particle acceleration by hydromagnetic turbulence. Astrophys. Space Sci. 12, 302 (1971).

Leamon, R. J., C. W. Smith, N. F. Ness, and H. K. Wong, Dissipation range dynamics: Kinetic Alfvén waves and the importance of $\beta_{e}$. J. Geophys. Res. 104, 22331 (1999).

Lee, M. A., and I. Lerche, Waves and irregularities in the solar wind. Rev. Geophys. Space Sci. 12, 671 (1974).

Levinson, A., Electron injection in collisionless shocks. Astrophys. J. 401, 73 (1992).

Lu, Q., and L. Chen, Ion heating by a spectrum of obliquely propagating low-frequency Alfvén waves. Astrophys. J. 704, 743 (2009).

Lysak, R. L., and W. Lotko, On the kinetic dispersion relation for shear Alfvén waves. J. Geophys. Res. 101, 5085 (1996).

Matsukiyo, S., and T. Hada, Relativistic particle acceleration in developing Alfvén turbulence. Astrophys. J. 692, 1004 (2009).

Matsumoto, H., Nonlinear whistler-mode interaction and triggered emissions in the magnetosphere, in Wave Instabilities in Space Plasmas, P. J. Palmadesso and K. Papadopoulos (eds). (D. Reidel, 1979).

Oka, M., T. Terasawa, Y. Seki, M. Fujimoto, Y. Kasaba, H. Kojima, I. Shinohara, H. Matsui, H. Matsumoto, Y. Saito, and T. Mukai, Whistler critical Mach number and electron acceleration at the bow shock: Geotail observation. Geophys. Res. Lett. 33, L24104 (2006).

Omura, Y., N. Furuya, and D. Summers, Relativistic turning acceleration of resonant electrons by coherent whistler mode waves in a dipole magnetic field. J. Geophys. Res. 112, A06236 (2007).

O'Sullivan, S., B. Reville, and A. M. Taylor, Stochastic particle acceleration in the lobes of giant radio galaxies. Mon. Not. R. astr. Soc. 400, 248 (2009).

Palmadesso, P., and G. Schmidt, Collisionless damping of a large amplitude whistler wave. Phys. Fluids 14, 1411 (1971). 
Petrosian, V., and S. Liu, Stochastic acceleration of electrons and protons. I. Acceleration by parallel-propagating waves. Astrophys. J. 610, 550 (2004).

Schlickeiser, R., Cosmic-ray transport and acceleration. I. Derivation of the kinetic equation and application to cosmic rays in static cold media. Astrophys. J. 336, 243 (1989a).

Schlickeiser, R., Cosmic-ray transport and acceleration. II. Cosmic rays in moving cold media with application to diffusive shock wave acceleration. Astrophys. J. 336, 264 (1989b).

Schlickeiser, R., Cosmic Ray Astrophysics. (Springer-Verlag Berlin Heidelberg New York, 2002).

Schlickeiser, R., and J. A. Miller, Quasi-linear theory of cosmic ray transport and acceleration: The role of oblique magnetohydrodynamic waves and transit-time damping. Astrophys. J. 492, 352 (1998).

Schmitt, J. P. M., Nonlinear theory of rf heating at cyclotron harmonics. Phys. Fluids 19, 245 (1976).

Shalchi, A., Nonlinear Cosmic Ray Diffusion Theories. (Springer-Verlag Berlin Heidelberg, 2009).

Skilling, J., Cosmic ray streaming. I. Effect of Alfvén waves on particles. Mon. Not. R. astr. Soc. 172, 557 (1975)

Smirnov, Y. N., and D. A. Frank-Kamenetskii, Nonlinearity and parametric resonance in a plasma. Sov. Phys. JETP. 26, 627 (1968).

Stix, T. H., Waves in Plasmas, (Springer-Verlag New York, 1992).

Summers, D., and C.-Y. Ma, A model for generating relativistic electrons in the Earth's inner magnetosphere based on gyroresonant wave-particle interactions. J. Geophys. Res. 105, 2625 (2000).

Tao, X., and J. Bortnik, Nonlinear interactions between relativistic radiation belt electrons and oblique whistler mode waves. Nonlin. Processes Geophys. 17, 599 (2010).

Terasawa, T., and M. Nambu, Ion heating and acceleration by magnetosonic waves via cyclotron subharmonic resonance. Geophys. Res. Lett. 16, 357 (1989).

Viratanen, J. J. P., and R. Vainio, Stochastic acceleration in relativistic parallel shocks. Astrophys. J. 621, 313 (2005)

Watanabe, Y., and T. Terasawa, On the excitation mechanism of the low-frequency upstream waves, J. Geophys. Res. 89, 6623 (1984).

White, R., L. Chen, and Z. Lin, Resonant plasma heating below the cyclotron frequency. Phys. Plasmas 9, 1890 (2002).

Winske. D., and M. M. Leroy, Diffuse ions produced by electromagnetic ion beam instabilities, J. Geophys. Res. 89, 2673 (1984). 